\title{
CRYOGENIC TESTS OF THE ATLAS LIQUID ARGON CALORIMETER
}

\author{
J. Bremer ${ }^{1}$, M. Chalifour ${ }^{2,3}$, C. Fabre ${ }^{1}$, A. Gonidec ${ }^{2}$ and G. Passardi ${ }^{1}$
}

The ATLAS liquid argon calorimeter consists of the barrel and two end-cap detectors housed in three independent cryostats filled with a total volume of $78 \mathrm{~m}^{3}$ of liquid argon. During cool-down the temperature differences in the composite structure of the detectors must be kept within strict limits to avoid excessive mechanical stresses and relative displacements. During normal operation the formation of gas bubbles, which are detrimental to the functioning of the detector, must be prevented and temperature gradients of less than $0.7 \mathrm{~K}$ across the argon bath are mandatory due to the temperature dependence of the energy measurements. Between April 2004 and May 2005 the barrel (120 t) and one end-cap (219 t) underwent qualification tests at the operating temperature of $87.3 \mathrm{~K}$ using a dedicated test facility at ground level. These tests provided a validation of the cooling methods to be adopted in the final underground configuration. In total $6.9 \mathrm{GJ}$ and $15.7 \mathrm{GJ}$ were extracted from the calorimeters and a temperature uniformity of the argon bath of less than $0.4 \mathrm{~K}$ was achieved.

1 CERN, Accelerator Technology department, Geneva, Switzerland

2 CERN, Physics department, Geneva, Switzerland

3 Also CEA/Saclay, Gif-sur-Yvette, France

Presented at the CEC-ICMC'05 Conference

29 August-2 September 2005, Keystone, Colorado, USA

Administrative Secretariat

AT Department

CERN

CH - 1211 Geneva 23
Geneva, Switzerland

12 January 2006 


\title{
CRYOGENIC TESTS OF THE ATLAS LIQUID ARGON CALORIMETER
}

\author{
J. Bremer ${ }^{1}$, M. Chalifour ${ }^{2,3}$, C. Fabre ${ }^{1}$, A. Gonidec ${ }^{2}$ and G. Passardi ${ }^{1}$ \\ ${ }^{1}$ Accelerator Technology department, CERN \\ 1211, Geneva 23, Switzerland \\ ${ }^{2}$ Physics department, CERN \\ 1211, Geneva 23, Switzerland \\ 3 also CEA/Saclay \\ Gif-sur-Yvette, 91191, France
}

\begin{abstract}
The ATLAS liquid argon calorimeter consists of the barrel and two end-cap detectors housed in three independent cryostats filled with a total volume of $78 \mathrm{~m}^{3}$ of liquid argon. During cool-down the temperature differences in the composite structure of the detectors must be kept within strict limits to avoid excessive mechanical stresses and relative displacements. During normal operation the formation of gas bubbles, which are detrimental to the functioning of the detector, must be prevented and temperature gradients of less than $0.7 \mathrm{~K}$ across the argon bath are mandatory due to the temperature dependence of the energy measurements. Between April 2004 and May 2005 the barrel (120 t) and one end-cap (219 t) underwent qualification tests at the operating temperature of $87.3 \mathrm{~K}$ using a dedicated test facility at ground level. These tests provided a validation of the cooling methods to be adopted in the final underground configuration. In total $6.9 \mathrm{GJ}$ and $15.7 \mathrm{GJ}$ were extracted from the calorimeters and a temperature uniformity of the argon bath of less than $0.4 \mathrm{~K}$ was achieved.
\end{abstract}

KEYWORDS: Liquid argon, cryogenic plant, ATLAS.

PACS: 07.20.Mc, 29.40.Vj 


\section{INTRODUCTION}

ATLAS [1] is one of the five particle detectors being constructed in order to study the interactions of proton or heavy-ion head-on collisions at the CERN Large Hadron Collider (LHC). The central part of the ATLAS detector, the liquid argon calorimeter, consists of the barrel and two end-cap detectors with cold masses of $120 \mathrm{t}$ and $2 \times 219 \mathrm{t}$. They are housed in three independent cryostats filled with $40 \mathrm{~m}^{3}$ and $2 \times 19 \mathrm{~m}^{3}$ of liquid argon, respectively.

Before final installation in the underground experimental cavern, the detectors undergo qualification tests at the operating temperature using a dedicated test facility in the assembly area. Between April 2004 and May 2005 the barrel and the end-cap C have been individually cooled down to liquid argon temperature and kept at this temperature for respectively, 10 and 7 weeks of testing.

\section{THE BARREL AND END-CAP LIQUID ARGON CALORIMETERS}

\section{The Barrel Calorimeter}

The barrel electromagnetic calorimeter (EM barrel) [2][3] consists of two identical half-wheels separated by a 4-mm gap (see FIGURE 1). Each wheel is divided in 16 modules, made of 64 accordion-shaped particle absorber plates, interleaved with copper electrodes. The absorbers are made of lead plates sandwiched between stainless-steel sheets, which are glued by resin-impregnated glass-fiber fabric. At the inner and outer edges, each absorber in encased in a G10 glass-epoxy composite precision bar. The outer G10 bars are fixed to 7 stainless-steel outer rings which support and give rigidity to the wheel. At the inner radius, the G10 bars are fixed to 8 composite inner rings which define the inner geometry of the wheel.

The presampler, built from thin glass-epoxy shells, acts as a thin active layer of liquid argon, which provides a first sampling of the electromagnetic showers in front of the EM barrel.

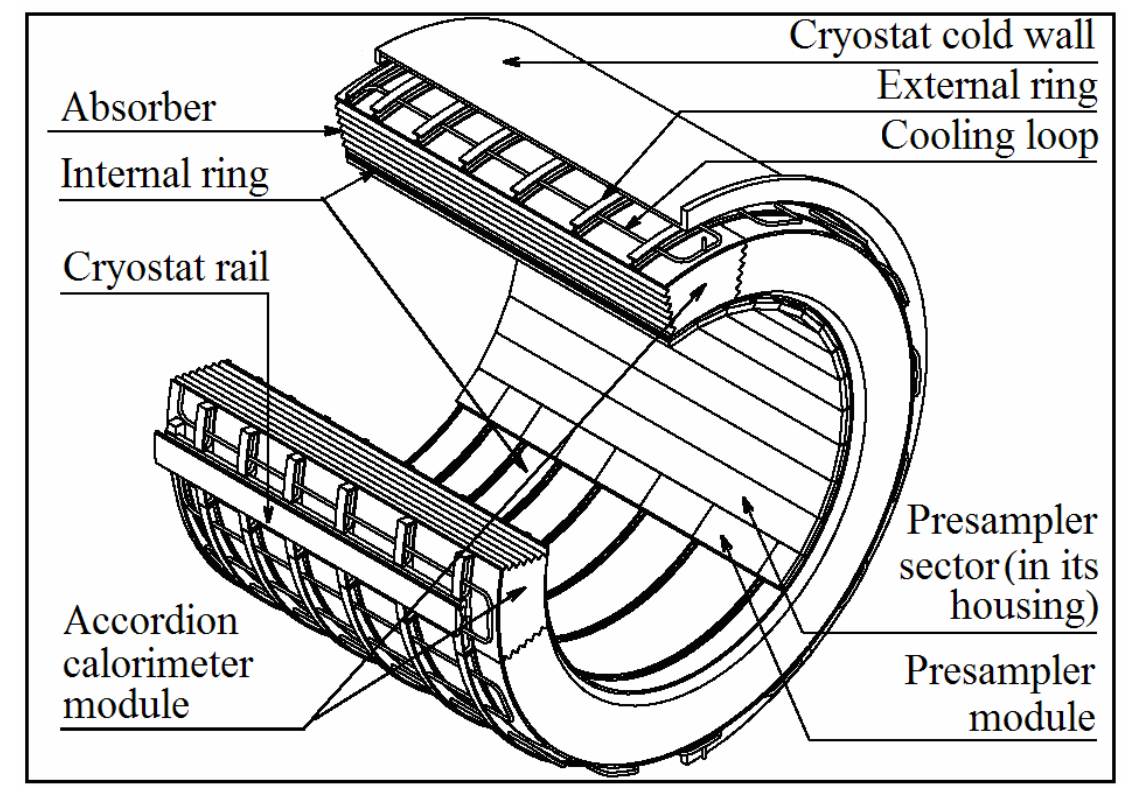

FIGURE 1. General layout of one barrel half-wheel. 
The electromagnetic barrel calorimeter is housed in the barrel cryostat made of aluminium. In addition, it supports and provides the vacuum for the central superconducting solenoid coil which generates the magnetic field for the inner tracker [4]. The warm vessel forms a hollow cylinder which contains a similarly shaped cold vessel. The outer cold cylinder is 6.5-m long and has a diameter of $4.3 \mathrm{~m}$. Each calorimeter wheel is supported on two aluminium sliding rails connected to the outer rings. The sliding rails are in turn posed on a counter rail connected to the cold vessel. The wheels and cryostat rails are only fixed together at the extremities of the cryostat. There are 32 signal and 1 high-voltage feedthroughs for each half-barrel distributed radially around each end of the cryostat. Each signal feedthrough brings a total of 2000 signal, monitoring and calibration cables through an independent insulation vacuum, out of the liquid argon bath. The barrel cryostat weights about $203 \mathrm{t}$ when filled with liquid.

Four heat exchangers made of 20/22-mm diameter stainless-steel pipe each with a length of about $60 \mathrm{~m}$ are integrated in the calorimeter outer rings. They are symmetrically distributed around each detector wheel: one at the upper half and one at the lower. One additional heat exchanger shaped in loops is placed at each cryostat extremity, close to the feedthroughs.

\section{The End-Cap Calorimeter}

As for the barrel, the end-cap cryostats are built out of aluminium, and are vacuum insulated. Each cryostat forms a hollow cylinder around the beam axis and has a $3 \mathrm{~m}$ long cold vessel with a diameter of $4.3 \mathrm{~m}$. It is equipped with 28 feedthroughs for signal and calibration cables and 2 high-voltage feedthroughs distributed radially around on the cryostat end. Two heat exchangers made of 20/22 mm diameter stainless-steel pipe each with a length of about $82 \mathrm{~m}$ are fixed on the outer cold cylinder: one at the upper half and one at the lower. Each end-cap contains an electromagnetic wheel (EMEC), two hadronic wheels (HEC1 and HEC2) and a forward calorimeter (FCAL) and weights, when full, about 269 t.

The EMEC is a complex structure of a composition similar to the barrel, except it consists of two concentric wheels supported by six support rings: three on the front and three on the back. While the intermediate front ring is made of a glass-epoxy composite material, the five others are made of aluminum.

The HEC1 and HEC2 are independent wheels divided into 32 identical modules built out of copper-plate absorbers alternating with electrodes and whose structural strength is maintained by stainless-steel tie rods. The modules are bolted together at the inner and outer radii. The HEC wheels sit on rails, shared with the EMEC, in the end-cap cryostats.

The FCAL is composed of one electromagnetic and two hadronic modules which rest in the cryostat forward tube. The first module is made of copper while the other two are of tungsten. Each of them consists of a single absorber matrix carrying an array of tube electrodes in holes in the matrix body.

\section{MAIN REQUIREMENTS FOR THE ASSOCIATED CRYOGENIC SYSTEM}

\section{Requirements during Cooling Process}

As shown in the previous section, the calorimeters are highly complicated structures made out of various materials having different thermal properties. During the cooling process the various detector components have different cooling rates and thermal 
contractions. Temperature differences thus induce mechanical stresses or relative displacements which can become unacceptable.

For instance, the two barrel wheels are supported on sliding rails fixed at the end of the cryostat rails. Under small temperature differences between the detector and cryostat rails, the two wheels could come closer to each other and may be damaged. Moreover, with the heat exchangers being integrated into the EM-barrel outer rings, the stainless steel rings cool down quicker than the G10 bars fixed to them, which in turn cool quicker than the inner rings. This may cause sagging of the bars and buckling of the absorbers. Regarding the end-cap calorimeter, the EMEC intermediate front ring, which is made out of composite material, and the absorbers have been identified as the most critical components, the main loads being induced by the radial shrinkage of the outer ring and the differential shrinkage of the front and rear outer rings.

Thermo-mechanical studies and tests [5][6] have been carried out by the various detector teams in order to define the maximum acceptable stresses and corresponding loads on the most critical components. These loads have been translated in term of maximum contractions and thus in term of maximum temperature differences between components. In total 6 temperature and 1 displacement criteria have been defined for the barrel and 11 temperature criteria for the end-cap. TABLE 2 summarizes the requirements to be met by the cryogenic system during the cooling process. Worth noticing is the temperature dependence of the EMEC criteria which become more stringent when cold.

\section{Requirements during Normal Operation}

During normal operation [2] the temperature of the liquid argon bath in the cryostats must be maintained constant at $87.3 \mathrm{~K}$ and the temperature gradient across the bath must be less than $0.7 \mathrm{~K}$. Maintaining the temperature of the liquid in sensitive parts of the calorimeters uniform and stable with time is extremely important since temperature variations directly affect energy measurements. Besides, the formation of gas bubbles, which are detrimental to the functioning of the detectors, must be prevented. Finally, the purity of the argon must be below 2 ppm (volume) of oxygen equivalent.

TABLE 2. The barrel and end-cap cooling criteria.

\begin{tabular}{ll} 
Barrel & End-Cap \\
\hline Distance between wheels $>3 \mathrm{~mm}$ & Temperature difference within cryostats $<45 \mathrm{~K}$ \\
\hline $\begin{array}{l}\text { Temperature difference between both rails on one } \\
\text { side and temperature of the wheel }<12 \mathrm{~K}\end{array}$ & $\begin{array}{l}\text { Temperature differences within FCAL, HEC1, } \\
\text { HEC2 }<20 \mathrm{~K}\end{array}$ \\
\hline $\begin{array}{l}\text { Temperature difference between a ring probe and } \\
\text { the closest module temperature }<20 \mathrm{~K}\end{array}$ & $\begin{array}{l}\text { Temperature difference within EMEC front face } \\
\text { and within EMEC rear face }<20 \mathrm{~K} \text { at } 293 \mathrm{~K} \text { and }< \\
6 \mathrm{~K} \text { at } 93 \mathrm{~K}\end{array}$ \\
\hline $\begin{array}{l}\text { EMEC rear-front temperature difference }<10 \mathrm{~K} \\
\text { Longitudinal gradient in each module }<40 \mathrm{~K}\end{array}$ & $\begin{array}{l}\text { Vertical and horizontal gradients in EMEC }<30 \mathrm{~K} \\
\text { at 293 K and }<12 \mathrm{~K} \text { at 93 K }\end{array}$ \\
\hline $\begin{array}{l}\text { Vertical gradient in each wheel }<30 \mathrm{~K} \\
\text { in EMEC }<10 \mathrm{~K}\end{array}$ \\
\hline $\begin{array}{l}\text { Temperature difference between both wheels } \\
<10 \mathrm{~K}\end{array}$ & $\begin{array}{l}\text { Global EMEC gradient }<35 \mathrm{~K} \text { at } 293 \mathrm{~K} \text { and } \\
<15 \mathrm{~K} \text { at } 93 \mathrm{~K}\end{array}$ \\
\hline
\end{tabular}




\section{COOLING OF THE BARREL AND END-CAP C AT THE TEST FACILITY}

A cryogenic test facility has been built to enable the individual testing, at the operating temperature, of each of the calorimeters before final installation in the ATLAS experimental area. The cryogenic system has been based on the principles of the system which will be used in the final underground configuration and whose detailed description was given in [7]. The successive cooling of the barrel and of one end-cap (ECC) took place respectively in spring and autumn, 2004. During the tests the cryostats were equipped with their final expansion vessel and heat exchangers regulation valve box. Each cryostat is connected via a cryogenic transfer-line to its dedicated expansion vessel located about $1.5 \mathrm{~m}$ above it. Each expansion vessel is equipped with a heat exchanger.

Before cooling down the calorimeters it was necessary to remove the impurities present in the cryostats and detectors. This was achieved by pumping the cold vessel with an oil-free pump and then filling with clean and dry nitrogen gas. This operation was repeated until the remaining traces of water and oxygen were respectively below $300 \mathrm{ppm}$ and $20 \mathrm{ppm}$. The last 4 cycles were done using argon gas for the barrel and helium gas for the end-cap.

A total of 232 (barrel) and 163 (ECC) temperature probes (PT100 platinum resistors) are located in the detectors, on the rings and rails. Additionally, for the barrel, 8 position monitors measure the distance of each wheel to the cryostat mid-plane. These probes, read by the detector control system, were used to monitor the various temperature differences and relative displacements during cool-down. The cooling criteria defined in TABLE 2 triggered a common interlock sent to the cryogenics control system to stop the cooling process.

From ambient temperature, the cooling process was first achieved by forced convection of gaseous nitrogen in the heat exchangers, whose inlet temperature was decremented. Since each cryostat needs to be cooled down only twice, it has been decided not to purchase any sophisticated external circulator and heat exchanger system. Liquid nitrogen was taken from a pressurized $40-\mathrm{m}^{3}$ storage tank, vaporized in a water heat exchanger, then passed through a regulated $2 \times 12.5-\mathrm{kW}$ electrical heater for fine setting of the gas temperature. This method allows to control the heat-exchangers' inlet temperature and to adjust its lowering in time. The heat was then extracted by the free convection of gaseous helium (argon) in the end cap (barrel) cryostat. Argon was preferred as the contact gas for the barrel instead of helium since the estimated cool-down time was acceptable (total energy to be extracted 6.9 GJ compared to 15.7 GJ for the end cap) and pumping of helium when cold could, in this way, be avoided. The pressure of the contact gas was maintained, during cool-down, at $1.3 \times 10^{5} \mathrm{~Pa}$ in the cryostats by pure gas supply from external sets of cylinders.

At about $140 \mathrm{~K}$ for the barrel and $120 \mathrm{~K}$ for the end-cap, the heat exchange became ineffective and the gas cooling was switched to a circulation of vaporizing liquid nitrogen in the heat exchangers. The liquid was supplied by the nitrogen tank to a $15 \mathrm{~m}^{3}$ nitrogen phase separator kept at $2.1 \times 10^{5} \mathrm{~Pa}$. The liquid-nitrogen circulation was driven by a Barber \& Nichols cryogenic pump providing a flow of up to $250 \mathrm{~g} / \mathrm{s}$. The pressure after the pump was regulated to $4.5 \times 10^{5}$ Pa by means of a control loop, returning part of the flow directly to the phase separator. The inlet valve of the heat exchangers was controlling the decrease of the detector temperature, while the outlet valve was regulating the nitrogen pressure. In order to avoid condensation of argon on the barrel heat exchangers the pressure in the heat exchangers was regulated to $3.3 \times 10^{5} \mathrm{~Pa}$ (88.9-K saturation), while the argon pressure had been lowered to about $1.15 \times 10^{5} \mathrm{~Pa}$ (88.5-K saturation). 
At the end of the cooling, from $100 \mathrm{~K}$ to $90 \mathrm{~K}$, a few hundred liters of argon were liquefied in order to homogenize the temperature inside the cryostats and ease the filling operation. For this, the pressure in the top heat exchangers was regulated to $3.5 \times 10^{5} \mathrm{~Pa}$ (89.6-K saturation) while the argon pressure in the cryostat was set to $1.35 \times 10^{5} \mathrm{~Pa}$ (90.1-K saturation). For the end-cap cryostat, 4 intermediate rinsing cycles were necessary in order to remove the helium and replace it with argon.

The liquid argon was then transferred into the cryostats via the bottom line and through a mechanical and Oxysorb filter in order to limit the pollution to an acceptable level. The $\mathrm{O}_{2}$-equivalent purity was checked on the cryostat volume before filling (less than $1 \mathrm{ppm}$ ), and after the filter during filling (about $0.3 \mathrm{ppm}$ ).

After filling, the gas pressure above the liquid surface in the expansion vessel was regulated to $1.25 \times 10^{5} \mathrm{~Pa}$ (89.3-K saturation), using the heat exchanger placed in the gaseous volume. In order to prevent the formation of gas bubbles, the temperature of the argon bath in the cryostats was lowered with respect to the temperature of the liquid in the expansion vessel thus creating a sub-cooled liquid. In steady-state operation, the cryostat heat-exchanger inlet valves controlled the mass flow so that dry-out of part of the heatexchanger length is prevented. The mass-flow regulation was based on information from a void-fraction meter placed at the outlet of the heat exchangers. The outlet valve regulated the pressure of the saturated nitrogen $\left(2.7 \times 10^{5} \mathrm{~Pa}\right.$ for the barrel and $2.6 \times 10^{5} \mathrm{~Pa}$ for the end-cap). Besides, the minimum pressure in the closed nitrogen circuit was set to $2.1 \times 10^{5} \mathrm{~Pa}$ (84.1-K saturation) in the phase separator, just above the argon triple point.

At the end of a testing period of 7 to 10 weeks, after emptying the cryostats of the liquid argon, the calorimeters were warmed-up using resistors placed on the bottom outer cold cylinder for the barrel and on the cryostat cold end plate for the end-cap. The contact gas in the cryostats was the same as for the cool-down. The warm-up process was limited by the same temperature and displacement criteria defined for the cool-down.

\section{RESULTS}

FIGURE 2 shows the variation, from cool-down to warm-up, of the detector mean temperatures and temperature gradient read on the cryogenics control system.

An average mass flow of $54 \mathrm{~g} / \mathrm{s}$ of gaseous nitrogen was necessary to cool down the barrel from $295 \mathrm{~K}$ to $140 \mathrm{~K}$, while a mean temperature difference of $46 \mathrm{~K}$ between the inlet and the outlet of the heat exchangers was achieved. A mean exchange power of $1.8 \mathrm{~kW}$, which results from the gas enthalpy variation under these conditions subtracting the cryostat estimated heat input [2], allowed the extraction of about 5.3 GJ at the average cooling rate of $0.19 \mathrm{~K} / \mathrm{h}$. From $140 \mathrm{~K}$ to $100 \mathrm{~K}, 27 \mathrm{~g} / \mathrm{s}$ of vaporized nitrogen were used on average for the extraction of about $1.1 \mathrm{GJ}$, with about $1.4 \mathrm{~kW}$ of cooling power. This power was corrected for the cryostat estimated heat load and for the measured heat leak into the external cryogenic system. A remaining $0.5 \mathrm{GJ}$ were removed from the barrel, in the argon condensation mode down to $90 \mathrm{~K}$, with $30 \mathrm{~g} / \mathrm{s}$ mean nitrogen consumption and $1.8 \mathrm{~kW}$ enhanced exchange power. From these figures one can calculate the barrel total heat capacity between $295 \mathrm{~K}$ and $90 \mathrm{~K}$ to about $6.9 \mathrm{GJ}$ which is very close to the initially calculated figure (7.2 GJ [2]).

In steady-state operation, the liquid argon bath in the barrel cryostat was regulated to an average temperature of $87.8 \mathrm{~K}$, while the expansion vessel was kept at $1.25 \times 10^{5} \mathrm{~Pa}$ (89.3-K saturation). A sub-cooling of 3.0 to $6.6 \mathrm{~K}$ below the equilibrium temperature was thus provided, depending on the height in the cryostat. The non-uniformity of the argon bath was found to be less than $0.4 \mathrm{~K}$, and the stability over time better than $0.1 \mathrm{~K}$. 

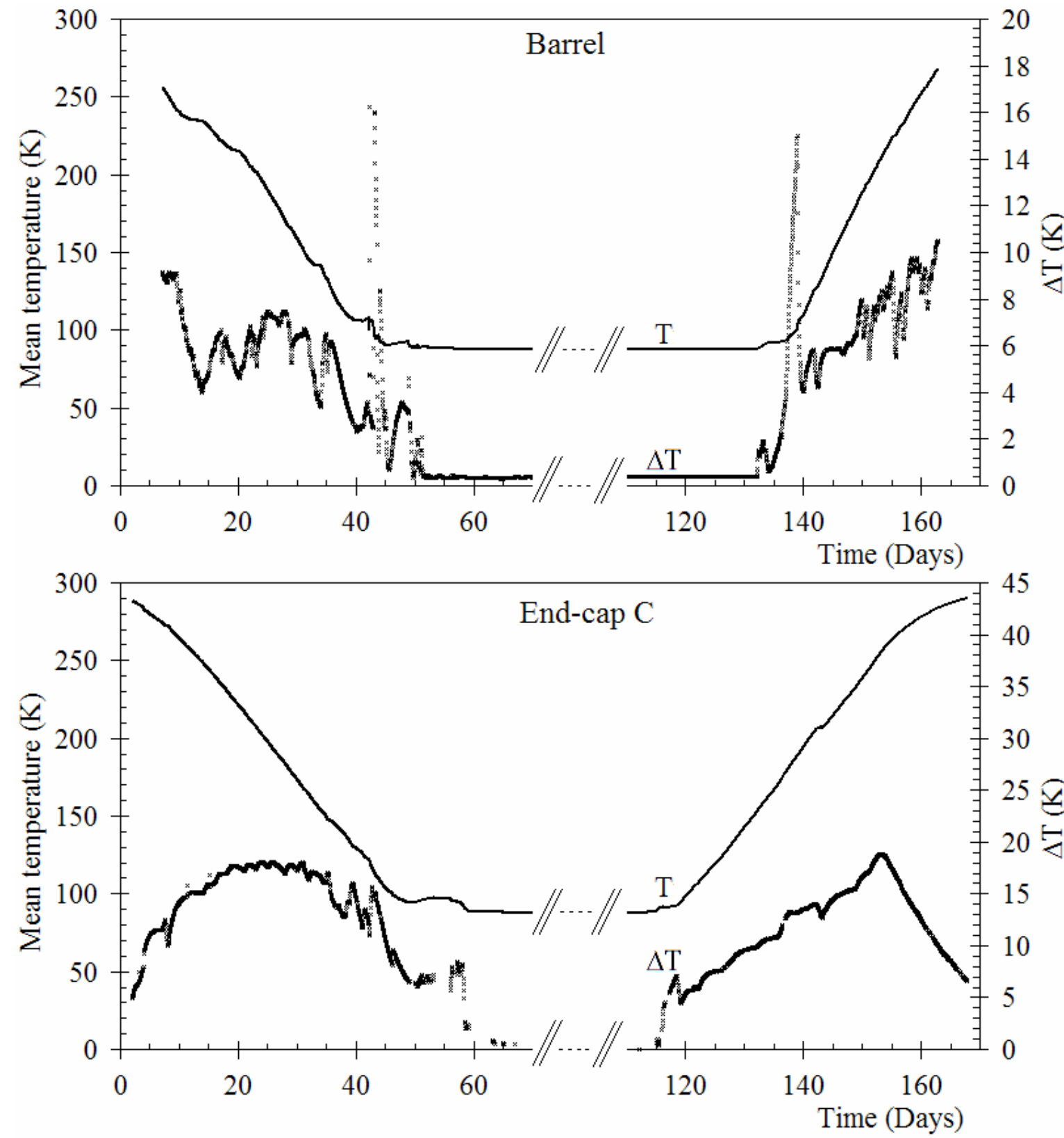

FIGURE 2. Variation, from cool-down to warm-up, of the barrel and end cap C mean temperature and temperature difference read on the cryogenics control system.

The evaporation of $22 \mathrm{~g} / \mathrm{s}$ of liquid nitrogen was necessary on average to maintain the temperature of the argon bath in stable condition. This corresponds to a total heat load to the barrel of $1.9 \mathrm{~kW}$, corrected for the external cryogenic system heat leak, which is in good agreement with the expected value $1.8 \mathrm{~kW}$ [2]. Note that the feedthroughs have an estimated contribution of $1 \mathrm{~kW}$, since they were individually heated during the complete process with 10 to $15 \mathrm{~W}$ in order to avoid condensation on the electronic connections.

From the end-cap cool-down data, and introducing similar power corrections for heat leaks as done for the barrel, one can assess the total heat capacity of the end-cap between $295 \mathrm{~K}$ and $90 \mathrm{~K}$ to about $15.7 \mathrm{GJ}$ also close to the $17.3 \mathrm{GJ}$ estimated in [2]. Indeed, the cool-down from $295 \mathrm{~K}$ to $120 \mathrm{~K}$ required an average gaseous nitrogen mass flow of $79 \mathrm{~g} / \mathrm{s}$, with a mean temperature difference of $49 \mathrm{~K}$ between the inlet and the outlet of the heat exchangers. An average cooling power of $3.5 \mathrm{~kW}$ thus allowed the extraction of around $13.0 \mathrm{GJ}$ from the end-cap with $0.17 \mathrm{~K} / \mathrm{h}$ as average cooling rate. Between $120 \mathrm{~K}$ and $100 \mathrm{~K}$ 
the evaporation of $37 \mathrm{~g} / \mathrm{s}$ provided about $4.0 \mathrm{~kW}$ to extract $1.4 \mathrm{GJ}$. The end of the cooling was achieved with an average liquid consumption of $22 \mathrm{~g} / \mathrm{s}$ for $1.3 \mathrm{~kW}$ exchange power and $1.3 \mathrm{GJ}$ removed.

In steady-state operation, the liquid-argon bath in the end-cap cryostat was regulated to an average temperature of $87.2 \mathrm{~K}$, while the expansion vessel was kept at $1.25 \times 10^{5} \mathrm{~Pa}$ (89.3-K saturation). A sub-cooling of 3.3 to $7.0 \mathrm{~K}$ below the equilibrium temperature was thus provided, depending on the height in the cryostat. The non-uniformity of the argon bath was found to be less than $0.4 \mathrm{~K}$, and the stability over time better than $0.2 \mathrm{~K}$.

The evaporation of $21 \mathrm{~g} / \mathrm{s}$ of liquid nitrogen was necessary on average to maintain the temperature of the argon bath. This corresponds to a measured heat load to the end-cap of $2.1 \mathrm{~kW}$ to be compared to the estimated value of $1.7 \mathrm{~kW}$ [2], which includes correction for the actual contribution of the HEC cold electronics depending on the fraction activated during the tests and for the additional anti-condensation heating introduced around the inner warm tube and on the warm end-plate.

Finally, for both cryostats the argon purity measured by purity monitors installed on the various detectors was between 0.1 and $0.2 \mathrm{ppm}$ of O2-equivalent.

\section{CONCLUSION}

The successful cool-down of the barrel and of the end-cap C calorimeters at the ground-level cryogenic test facility validated the cooling method based on a forced convection of gaseous nitrogen in the heat exchangers. This method permitted the extraction of about $6.9 \mathrm{GJ}$ from the barrel and $15.7 \mathrm{GJ}$ from the end-cap at the average cooling rates of $0.18 \mathrm{~K} / \mathrm{h}$ and $0.15 \mathrm{~K} / \mathrm{h}$, while limiting the temperature gradients in the calorimeters to acceptable values. Moreover, the achieved temperature uniformity and stability in the argon bath was fully satisfactory. The next important step is the cool-down of the barrel in the ATLAS final underground configuration at the end of 2005.

\section{ACKNOWLEDGEMENTS}

This work is the result of collaboration between BNL, CERN, LAL and LPSC.

\section{REFERENCES}

1. ATLAS Collaboration, Technical Proposal for a General-Purpose pp Experiment at the Large Hadron Collider at CERN, CERN LHCC-94-043, LHCC-P2 (1994), CERN.

2. ATLAS Collaboration, Liquid Argon Calorimeter Technical Design Report, CERN LHCC-96-041, ATLAS TDR 2 (1996), CERN.

3. ATLAS Electromagnetic Liquid Argon Calorimeter Group, "Construction, Assembly and Tests of the ATLAS Electromagnetic Barrel Calorimeter,” in Nucl. Instrum. Methods, (2005), to be published.

4. Doy, Y., et.al., "Final Testing of the ATLAS Central Solenoid before Installation," in Proceedings of the 20th International Cryogenic Engineering Conference (ICEC 20), Beijing, China 2004, to be published.

5. Chalifour, M., et.al., "Studies for Cooling Down the ATLAS Electromagnetic Barrel Calorimeter," ATLAS note EDMS ATL-AB-EN-0029, 2003.

6. Karst, P., et.al., "Specification for the Cooling and Heating of the EMEC," ATLAS note EDMS ATLAE-ES-0020, 2004.

7. Bremer, J., et.al., “The Cryogenic System for the ATLAS Liquid Argon Detector,” in Proceedings of the 18th International Cryogenic Engineering Conference (ICEC 18), Mumbai, India 2000, edited by K.G. Narayankhedkar, Narosa, New Delhi, 2000, pp. 219-222. 